\title{
Prediction of chain length effects in elongational flows of dilute polymer solutions by successive fine graining
}

\author{
P. Sunthar* J. Ravi Prakash*
}

(received 25 October 2004, revised 8 March 2005)

\begin{abstract}
A new computational tool for predicting the rheological properties of a dilute solution of polymers in $\theta$-conditions is presented. Within this approach, the polymer is modelled as a bead spring chain with finitely extensible springs and fluctuating hydrodynamic interactions incorporated. The novelty of the method lies in obtaining predictions for a very large Kuhn step chain by extrapolating results of a series of bead-springs representations to the bead-rod limit. This provides the computational advantage of using smaller number of modes in a coarse grained description and better accuracy in the extrapolated result. The effect of chain length in the unraveling dynamics of a polymer in elongational flow is examined using this approach.
\end{abstract}

${ }^{*}$ Department of Chemical Engineering, Monash University, Melbourne, Victoria 3800, Australia. mailto:Ravi. Jagadeeshan@eng.monash. edu.au

See http://anziamj.austms.org.au/V46/CTAC2004/Sunt for this article, (c) Austral. Mathematical Soc. 2005. Published May 3, 2005. ISSN 1446-8735 


\section{Contents}

1 Introduction

C321

2 Bead-spring chain model

C323

3 Method of successive fine graining

C325

3.1 Model parameters for a given $N \ldots \ldots \ldots$ C $\ldots \ldots \ldots$

3.2 Extrapolation of results . . . . . . . . . . . . . C327

4 Successive fine graining in elongational flow: Results

C328

5 Conclusion

C332

References

C333

\section{Introduction}

Many of the transport properties of dilute solutions of long chain polymer molecules are of industrial interest. Chief among them are rheological properties such as the shear and elongational viscosities. For instance, unlike a dilute suspension of rigid particles, the elongational (or extensional) viscosity of a solution of long and flexible molecules is orders of magnitude greater than that of its Newtonian solvent. Equilibrium and near equilibrium (or linear viscoelastic) properties of polymer solutions have been studied for long using simple coarse grained physical models for the polymer molecule $[13,16]$. While these fundamental models, such as the bead-spring chain model, currently employed are still the same, the regimes of application, namely strong flows, makes it difficult to obtain closed form analytical approximations. A commonly employed tool to overcome this difficulty is to use a Brownian Dynamics simulation (BDS) to numerically evaluate the consequences of the model under various flows. In this paper we present a computational strategy, 
in the context of BDS, which makes it easier to handle very large molecular weight polymers, and employ it to study the consequences of increasing polymer chain length in elongational flow.

The characteristic physical feature of a polymer molecule is its mechanical flexibility over several length scales beginning at distances of a few monomer units. This is commonly modelled as a chain of $N_{\mathrm{k}}$ rods which freely rotates about its joints. Each joint consists of a spherical bead representing the hydrodynamic drag felt by the rods in the solvent media. A typical polymer of a few million molecular weight would be modelled with a few thousand units in this bead-rod framework [1]. Due to the collisions of the surrounding solvent molecules, the polymer constantly changes its configuration and the movement of one bead influences that of the other beads by hydrodynamic interactions (HI) mediated through the solvent. While some studies neglect $\mathrm{HI}$ in the model to keep it simple and obtain certain gross and limiting behaviours [3], it is necessary to incorporate HI to explain the molecular weight scaling of dynamic properties at equilibrium and in flow. From a computational view point this translates to the computational time scaling as $N_{\mathrm{k}}^{4.5}$. This makes it currently difficult to simulate large molecular weight polymers with a bead rod model.

An approximation frequently employed to overcome this computational difficulty is to perform a coarse gaining in which segments of rods are replaced by springs which mimic the entropic resistance of the segments to stretching $[1,2]$. The simplest of such models is the dumbbell model in which the whole polymer is replaced by a single spring and two beads. However, this simplification does not capture the flow induced dynamics of the polymer arising due to its flexibility. Often a chain of $N$ beads connected by $N-1$ springs is used. This considerably reduces computational time with the number of beads reduced from a few thousands in the bead-rod model to few tens in the bead-spring model.

One of the main concerns of this paper is addressing the problem of how to systematically increase the number of springs in the chain. We show 
that by accumulating data in the region of small $N$, which can be easily computed, a suitable extrapolation can be carried out to obtain the limiting behaviour as $N \rightarrow N_{\mathrm{k}}$, thereby avoiding computations with a large $N_{\mathrm{k}}$ beadrod model. The procedure is developed in the context of the unraveling of the polymer molecule under extensional flow from a coiled state to a stretched state. Using this procedure we examine the effect of increasing $N_{\mathrm{k}}$ and its limiting behaviour as $N_{\mathrm{k}} \rightarrow \infty$. The model, governing equations, and the BDS scheme are outlined in Section 2. The method of successive fine graining is presented in Section 3 and the results of the effect of increasing $N_{\mathrm{k}}$ on the polymer conformations in elongational flow is discussed in Section 4

\section{Bead-spring chain model}

The dilute solution of polymer molecules is modelled as an ensemble of noninteracting bead-spring chains, each of which has $N$ beads. With $\psi\left(\mathbf{r}_{1}^{*}, \ldots, \mathbf{r}_{N}^{*}\right)$ being the configurational distribution function of the bead positions $\mathbf{r}_{\mu}^{*}$, the starting point in any statistical theory of bead-spring chains is given by the "diffusion" equation or Fokker-Planck equation for the evolution of $\psi[1,2,9]$ :

$$
\begin{aligned}
\frac{\partial \psi}{\partial t^{*}}=\sum_{\mu, \nu=1}^{N} \frac{\partial}{\partial \mathbf{r}_{\mu}^{*}} \cdot \boldsymbol{\Gamma}_{\mu \nu}\left(h^{*}\right) & \cdot \frac{\partial \psi}{\partial \mathbf{r}_{\nu}^{*}} \\
& -\sum_{\mu=1}^{N} \frac{\partial}{\partial \mathbf{r}_{\mu}^{*}} \cdot\left[\boldsymbol{\kappa}^{*} \cdot \mathbf{r}_{\mu}^{*}+\sum_{\nu=1}^{N} \boldsymbol{\Gamma}_{\mu \nu}\left(h^{*}\right) \cdot \mathbf{F}_{\nu}^{\mathbf{s}}(b)\right] \psi,
\end{aligned}
$$

Here, the lengths have been scaled by $l_{\mathrm{H}} \equiv \sqrt{k T / H}$, time by $\lambda_{\mathrm{H}} \equiv \zeta / 4 H$, and forces by $\sqrt{H k T}$, where $H$ is the linear spring constant, $k$ is Boltzmann's constant, $T$ is the temperature and $\zeta$ is the Stokes friction (drag) coefficient associated with the spherical bead of radius $a$. The HI parameter

$$
h^{*} \equiv \frac{a}{\sqrt{\pi} l_{\mathrm{H}}} .
$$


In Equation (1), $\boldsymbol{\kappa}^{*}$ is a time-dependent, homogeneous, velocity gradient tensor of the surrounding fluid motion, and $\boldsymbol{\Gamma}_{\mu \nu}$ is the hydrodynamic interaction tensor, representing the effect of the motion of a bead $\mu$ on another bead $\nu$ through the disturbances carried by the surrounding fluid,

$$
\Gamma_{\mu \nu}=\delta_{\mu \nu} \boldsymbol{\delta}+\boldsymbol{\Omega}_{\mu \nu}
$$

where, $\boldsymbol{\delta}$ and $\delta_{\mu \nu}$ represent a unit tensor and a Kronecker delta, respectively. The tensor $\Omega_{\mu \nu}$ is a function of the bead separation, $\Omega_{\mu \nu}=\Omega\left(\mathbf{r}_{\mu \nu}^{*}\right)$, for which we employ the Rotne-Prager-Yamakawa regularisation of the diffusion tensor $[12,6]$,

$$
\boldsymbol{\Omega}\left(\mathbf{r}^{*}\right)=\left[\Omega_{1} \boldsymbol{\delta}+\Omega_{2} \frac{\mathbf{r}^{*} \mathbf{r}^{*}}{r^{* 2}}\right]
$$

where, for $r^{*} \geq 2 \sqrt{\pi} h^{*}$,

$$
\Omega_{1}=\frac{3 \sqrt{\pi}}{4} \frac{h^{*}}{r^{*}}\left(1+\frac{2 \pi}{3} \frac{h^{* 2}}{r^{* 2}}\right), \quad \Omega_{2}=\frac{3 \sqrt{\pi}}{4} \frac{h^{*}}{r^{*}}\left(1-2 \pi \frac{h^{* 2}}{r^{* 2}}\right),
$$

and for $0<r^{*} \leq 2 \sqrt{\pi} h^{*}$,

$$
\Omega_{1}=1-\frac{9}{32} \frac{r^{*}}{h^{*} \sqrt{\pi}}, \quad \Omega_{2}=\frac{3}{32} \frac{r^{*}}{h^{*} \sqrt{\pi}} .
$$

Continuing with Equation (1), $\mathbf{F}_{\nu}^{\mathrm{s}}$ is the entropic spring force on bead $\nu$ due to adjacent beads, $\mathbf{F}_{\nu}^{\mathrm{s}}=\mathbf{F}^{\mathrm{c}}\left(\mathbf{Q}_{\nu}^{*}\right)-\mathbf{F}^{\mathrm{c}}\left(\mathbf{Q}_{\nu-1}^{*}\right)$, where $\mathbf{F}^{\mathrm{c}}\left(\mathbf{Q}_{\nu-1}^{*}\right)$ is the force between the beads $\nu-1$ and $\nu$, acting in the direction of the connector vector between the two beads $\mathbf{Q}_{\nu}^{*}=\mathbf{r}_{\nu}^{*}-\mathbf{r}_{\nu-1}^{*}$. The entropic spring force of a chain of freely jointed bead-rods is often approximated by the Warner spring [1]:

$$
\mathbf{F}_{\mathrm{FENE}}^{\mathrm{c}}\left(\mathbf{Q}^{*}\right)=\mathbf{Q}^{*}, \frac{1}{1-q^{* 2}},
$$

where $q^{*}=Q^{*} / \sqrt{b}$ is the ratio of the magnitude of the connector vector $Q^{*}$ and the fully stretched length $\sqrt{b}$. We ignore solvent quality effects. 
The exact solution of the time evolution of various averages carried out with the distribution function $\psi$, without any further approximations, can be obtained by writing a stochastic differential equation (SDE) equivalent to Equation (1) and solving it with the help of a Brownian dynamics algorithm [9]. In the presence of fluctuating HI, the problem of the computational intensity of calculating the Brownian term has been attenuated significantly recently [6] by the use of a Chebyshev polynomial representation [4] for the Brownian term. We adopt this strategy, and the details of the exact algorithm followed here are given in [10].

\section{Method of successive fine graining}

The key idea behind the method of successive fine graining (SFG) in the context of BDS under $\theta$-conditions comes from a recent study by Kröger et al. [7], which showed that finite $N$ data can be extrapolated to $N \rightarrow \infty$, to obtain predictions which are independent of the choice HI parameter $h^{*}$. We carry out a similar procedure under non-equilibrium conditions. Once the model parameters are identified at a given level of the bead-spring representation $N$, the polymer is simulated in the presence of flow at various values of $N$ and the accumulated data is then extrapolated in a similar manner. This section briefly outlines how the model parameters may be identified at a given level of bead-spring representation of the bead-rod chain, and how the data is to be extrapolated.

\subsection{Model parameters for a given $N$}

The key parameters in the model are the number of beads $N$, the finite extensibility parameter $b$ and the hydrodynamic interaction parameter $h^{*}$. In this work we restrict discussions to non-dimensional predictions, therefore we do not explicitly identify the scale parameters $l_{\mathrm{H}}$ and $\lambda_{\mathrm{H}}$. One way to 
choose the parameters is such that the model reproduces certain equilibrium properties of the real molecule. Here we distinguish between static and dynamic properties. The HI parameter does not alter static properties, which are purely determined by $N$ and $b$. As an example, the radius of gyration

$$
R_{\mathrm{g}, \text { mod }}^{* 2}=\chi^{2}(b) \frac{N^{2}-1}{2 N},
$$

where $\chi(b)$ is a known function of $b$ for a given spring force model. For Warner FENE springs it is

$$
\chi^{2}(b)=\frac{b}{b+5} .
$$

The contour length of the chain is

$$
L_{0, \bmod }=(N-1) \sqrt{b} .
$$

The number of Kuhn steps $N_{\mathrm{k}}$ in the real polymer is

$$
N_{\mathrm{k}} \equiv \frac{L_{0, \exp }^{2}}{6\left(R_{\mathrm{g}, \exp }^{\theta}\right)^{2}}
$$

where $L_{0, \exp }$ is the measured contour length and $R_{\mathrm{g}, \exp }^{\theta}$ is the measured radius of gyration. Constructing a similar ratio from the model expressions from Equations (8) and (10) we have

$$
\frac{b}{\chi^{2}(b)}=N_{\mathrm{k}} \frac{3(N+1)}{N(N-1)} .
$$

Given the experimentally measured ratio $N_{\mathrm{k}}$ and an arbitrary choice of the number of beads $N$, the parameter $b$ is evaluated from Equation (12). The determination of $h^{*}$ for a given choice of $N$ and $b$ so that the model reproduces the dynamical equilibrium properties is a difficult problem in general [15]. However, in the long chain limit $N_{\mathrm{k}} \rightarrow \infty$, it can be shown that the dynamical properties are insensitive to the exact value of the HI parameter $h^{*}$ [7]. We 
have shown [15] that in the case of finitely extensible springs the correct parameter that characterises HI is given by $\tilde{h}^{*}=h^{*} / \chi$ for a given value of $b$. An arbitrary value is assigned for $\tilde{h}^{*}$. Physically meaningful values of $\tilde{h}^{*}$ lie in the range $0<\tilde{h}^{*} \lesssim 0.5[8]$.

Another crucial parameter that arises only in the presence of flow is the Weissenberg number, Wi. In this work

$$
\mathrm{Wi}=\dot{\epsilon} \lambda_{1},
$$

where $\dot{\epsilon}$ is the elongation rate and $\lambda_{1}$ is the longest relaxation time, evaluated by fitting an exponential to the tail of the relaxation of the function $\bar{x}^{2}(t)$, where $\bar{x}(t)$ is the average instantaneous stretch, when a fully stretched polymer is left to relax to equilibrium as it is done in experiments of fluorescently dyed polymers [14]. In terms of a bead-spring model the "stretch" or "extension" is defined as, $x_{\max } \equiv \max _{\mu, \nu}\left|r_{\mu}^{x}-r_{\nu}^{x}\right|$, where $r_{\mu}^{x}$ is the $x$-component of vector $\mathbf{r}_{\mu}$, with $x$ being the flow direction. Here, the average along the direction of intended elongation is denoted as $\bar{x} \equiv\left\langle x_{\max }\right\rangle$.

\subsection{Extrapolation of results}

In the presence of flow, in addition to using a model with finitely extensible springs (FES), the total number of springs considered must also be finite, so that the consequences of the finite size of the molecule may be accurately represented. Simulations of bead-spring chain models with FES in extensional flows have shown that, in general, in order to predict the behavior accurately across a wide range of regimes, it is necessary to use models with a large number of springs [5]. In principle, the maximum number of springs, $(N-1)$, that one can use, is equal to the number of Kuhn steps in the underlying chain. In practice, with the force models currently used, FENE or WLC, it is not possible to reach $(N-1) \rightarrow N_{\mathrm{k}}$. This is because of the limitations imposed by the nature of $\chi(b)$ in Equation (12). It is straightforward to see, 
for instance, in the case of FENE springs, where, $\chi(b)=\sqrt{b /(b+5)}$, that $b$ becomes negative near $N=3 N_{\mathrm{k}} / 5$.

The central hypothesis of the present work is that the behavior of an underlying chain with $N_{\mathrm{k}}$ Kuhn steps, may be predicted by accumulating data for a bead-spring chain model with FES, at various values of $N$ where the force law is valid, and extrapolating the accumulated results to the limit $N \rightarrow$ $N_{\mathrm{k}}$. Since increasing values of $N$ represent more fine-grained versions of the underlying chain, the procedure is called "successive fine-graining."

For a polymer with fixed contour length (or equivalently, a fixed value of $N_{\mathrm{k}}$ ), successive fine-graining implies that the parameter $b$ becomes a function of $N$, given by Equation (12). Furthermore, during the SFG procedure, the value of $\tilde{h}^{*}$ is held constant as $N \rightarrow N_{\mathrm{k}}$. Physically, this is equivalent to assuming that the hydrodynamic interaction parameter at each level of the fine graining process is constant and equal to that of the underlying chain. (Note that the conventional HI parameter $h^{*}=\chi \tilde{h}^{*}$, changes as $b$ changes).

\section{Successive fine graining in elongational flow: Results}

The SFG procedure outlined above is now applied to case of a dilute solution of polymers undergoing elongational flow. In this work we focus our attention on the prediction of size measures. The prediction of elongational viscosity is treated elsewhere [11]. The conformation of the polymer is characterised by the expansion factor

$$
E=\frac{\bar{x}^{+}}{\bar{x}}
$$

which is the ratio of the transient stretch to its value at equilibrium. This quantity is experimentally accessible when the polymers are visually observed under a microscope as they undergo expansion in elongational flow [14]. In 
an earlier work it was shown that it is possible to accurately predict the conformations of DNA using the SFG procedure [15]. The effect of increasing polymer chain length is examined here. Polymers of three different lengths are considered, namely, $N_{\mathrm{k}}=200,600$ and 2000. The limiting behaviour for $N_{\mathrm{k}} \rightarrow \infty$ is also given.

For polymers with finite $N_{\mathrm{k}}$, data is gathered at finite values of $N=$ $15,17,19,23,27$ and extrapolated to the corresponding $N \rightarrow N_{\mathrm{k}}$ limit. At each value of $N$, the parameters $b$ and $h^{*}$ required for the simulation are obtain as discussed in Section 3.1. Two values of $\tilde{h}^{*}$ are considered: $\tilde{h}^{*}=$ 0.19 , and 0.3 , which bracket the fixed point value of 0.24 (with pre-averaged HI) [15]. Once the longest relaxation time is estimated, the flow elongational rates are fixed according to Equation (13). A Weissenberg number of Wi $=2$ is considered. In all the simulations considered below, averages were obtained using an ensemble of $10^{4}$ polymer molecules.

An illustration of the extrapolation of finite $N$ data to $N \rightarrow N_{\mathrm{k}}$ is shown in Figure 1 for $N_{\mathrm{k}}=600$. At each value of the strain $\epsilon=\dot{\epsilon} t$, the value of expansion factor $E$ obtained from the finite $N$ runs is extrapolated to $N \rightarrow$ 600 . The locus of all extrapolated points at various strains for the two chosen values of $\tilde{h}^{*}$ is given in Figure 2. The two curves are indistinguishable within error bars for small strain units and begin to come apart with increasing strain. The physical reason for this is explained elsewhere [15].

In Figure 3, the unraveling of polymers of various chain lengths are compared. Also shown is the limiting behaviour for $N_{\mathrm{k}} \rightarrow \infty$, which is the universal unraveling of a polymer under $\theta$-conditions obtained by using a Hookean spring force model and extrapolating the finite $N$ data to the limit $N \rightarrow \infty$ [15]. In Figure 3, the expansion factor $E$ for the finite $N_{\mathrm{k}}$ polymers is seen to level off to an asymptotic steady state value, clearly highlighting the central difference between finite and infinite chains. At a threshold value of strain, the finite character of the chain leads to the existence of a point of inflection on the curves, where the slope stops increasing and starts to decrease. The point of inflexion occurs at higher strains for longer chains. 


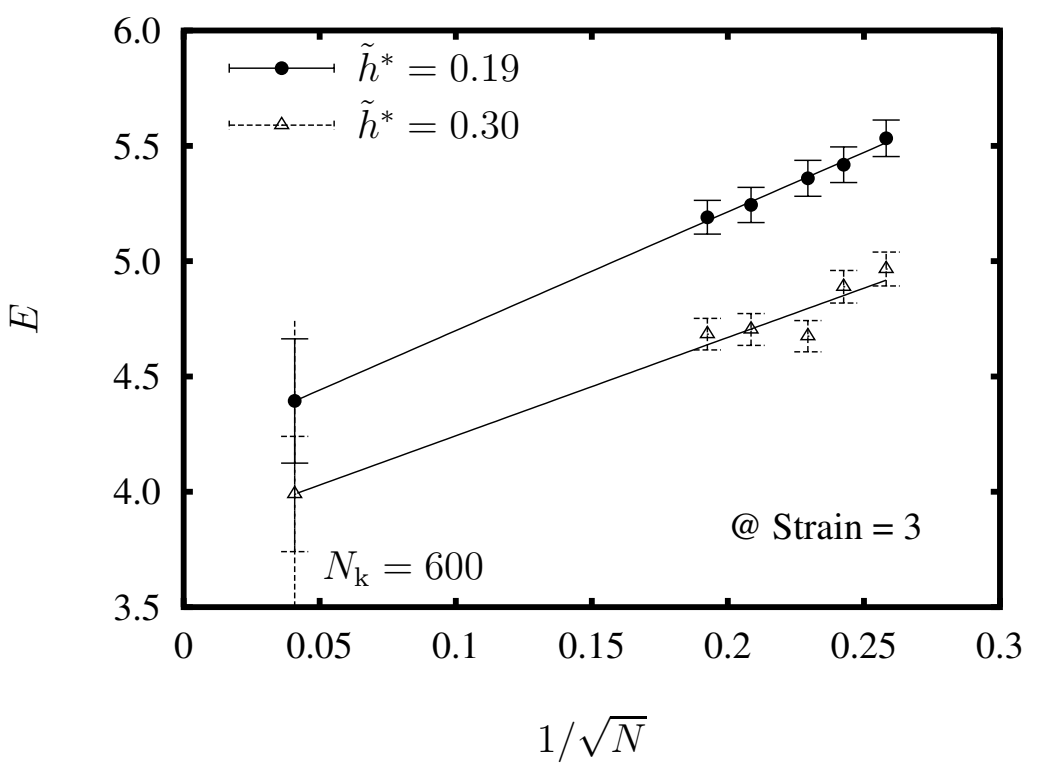

FIGURE 1: Illustration of the SFG procedure for a model with FENE springs, at a fixed value of Hencky strain, $\epsilon=3$ for $N_{\mathrm{k}}=600$. Symbols for $N<N_{\mathrm{k}}$ have been obtained by carrying out simulations at two different values of $\tilde{h}^{*}$. Lines through the symbols represent an extrapolation to the limit $N \rightarrow N_{\mathrm{k}}$. 


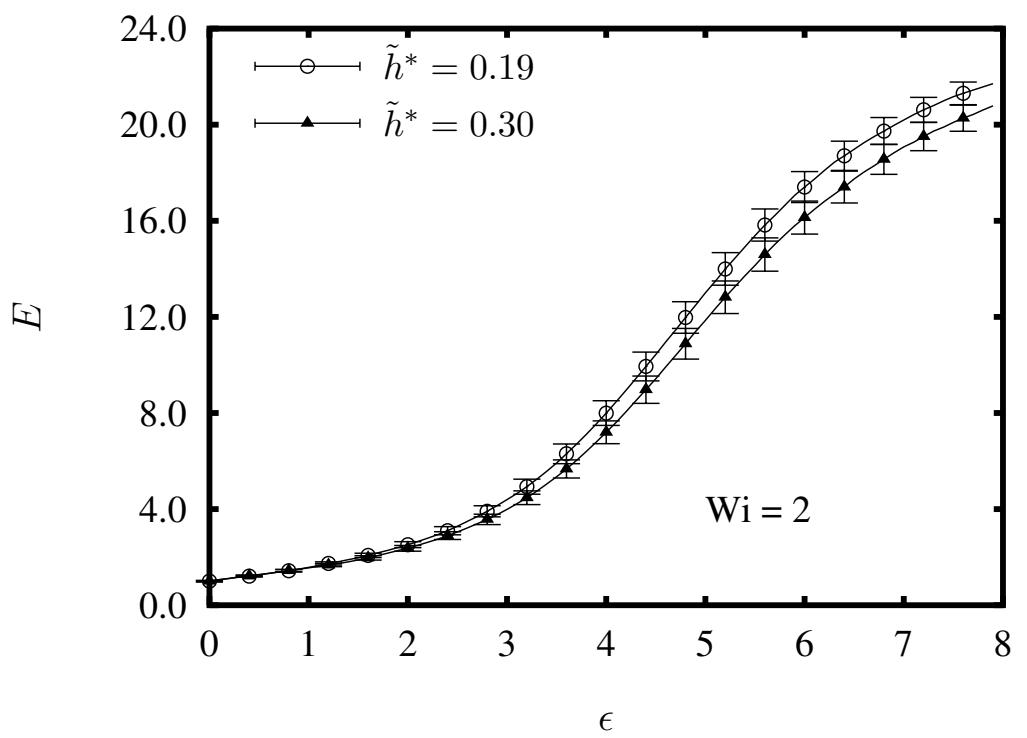

FiguRE 2: Expansion factor $E$, in extensional flow, for a model with FENE springs with two different values of $\tilde{h}^{*}$. Symbols are extrapolated values, obtained by the SFG procedure with $N_{\mathrm{k}}=600$. 


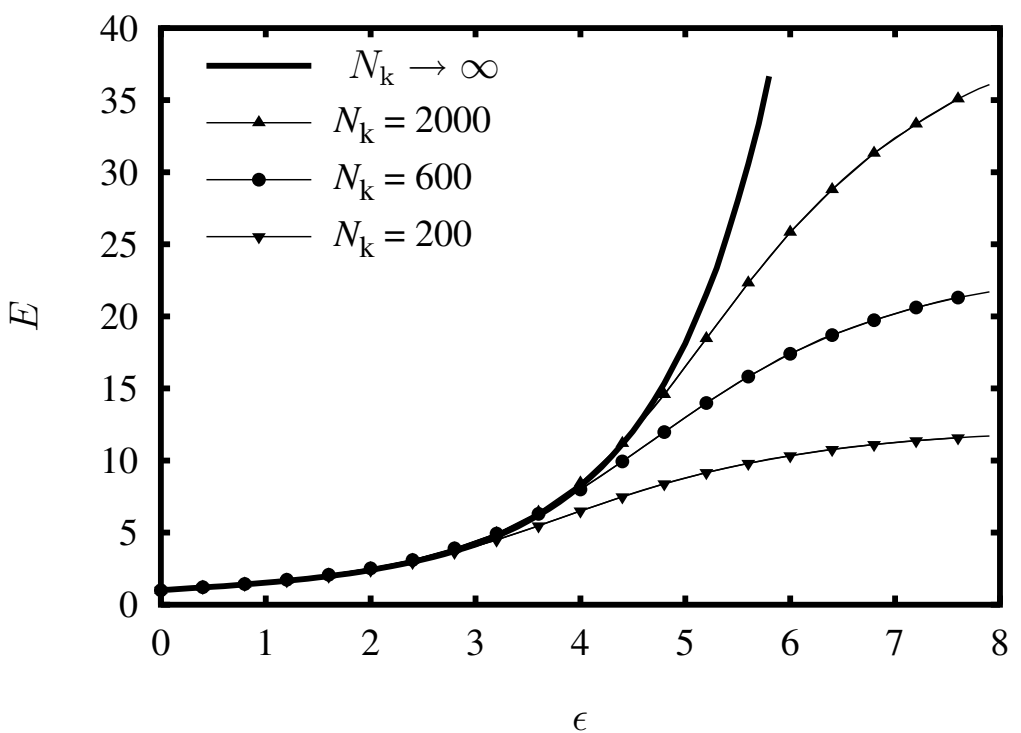

FiguRE 3: Effect of chain length on the unraveling of a polymer in elongational flow. The HI parameter is $\tilde{h}^{*}=0.19$ and $\mathrm{Wi}=2$, in all cases.

This corroborates the expectation that longer chain lengths remain in a coil like state for larger strains before the finiteness is explored by the flow.

\section{Conclusion}

The effect of polymer chain length on the unraveling dynamics in elongational flow was examined using the method of successive fine graining (SFG). The method provides a unified framework to study both chains of finite lengths and its limiting behaviour at an infinite lengths. It was shown that the unraveling curve of infinite length polymer acts as an envelope for all finite chains. Essentially, for a given value of the Weissenberg number, the strain 
at which a finite chain curve departs from the Hookean curve, depends on the value of $N_{\mathrm{k}}$. For increasing values of $N_{\mathrm{k}}$, the point of departure occurs at increasing values of strain. The SFG method also provides a computationally tractable procedure to model very long chain polymer molecules, which are otherwise difficult to be treated with the bead-rod model. Predictions for a chain with $N_{\mathrm{k}}$ as large as 2000 rods was obtained with bead-spring chains with as few as 27 beads.

Acknowledgment: This work has been supported by a grant from the Australian Research Council, under the Discovery Projects program.

\section{References}

[1] R. B. Bird, C. F. Curtiss, R. C. Armstrong, and O. Hassager. Dynamics of Polymeric Liquids - Volume 2: Kinetic Theory. John Wiley, New York, second edition, 1987. C322, C323, C324

[2] M. Doi and S. F. Edwards. The Theory of Polymer Dynamics. Clarendon Press, Oxford, New York, 1986. C322, C323

[3] P. S. Doyle and E. S. G. Shaqfeh. Dynamic simulations of freely draining, flexible bead-rod chains: Start-up of extensional and shear flow. J. Non-Newtonian Fluid Mech., 76:43-78, 1998. http://dx.doi.org/10.1016/S0377-0257(97)00112-2. C322

[4] M Fixman. Construction of Langevin forces in the simulation of hydrodynamic interaction. Macromolecules, 19:1204-1207, 1986. http://dx.doi.org/10.1021/ma00158a043. C325

[5] C.-C. Hsieh, L. Li, and R. G. Larson. Modeling hydrodynamic interaction in Brownian dynamics: Simulations of extensional flows of 
dilute solutions of DNA and polystyrene. J. Non-Newtonian Fluid Mech., 113:147-191, 2003.

http://dx.doi.org/10.1016/S0377-0257(03)00107-1. C327

[6] R. M. Jendrejack, M. D. Graham, and J. J. de Pablo. Hydrodynamic interactions in long chain polymers: Application of the Chebyshev polynomial approximation in stochastic simulations. J. Chem. Phys., 113(7):2894-2900, 2000. http://dx.doi.org/10.1063/1.1305884. C324, C325

[7] M. Kröger, A. Alba-Pérez, M. Laso, and H. C. Öttinger. Variance reduced Brownian simulation of a bead-spring chain under steady shear flow considering hydrodynamic interaction effects. J. Chem. Phys., 113(11):4767-4773, 2000.

http://dx.doi.org/10.1063/1.1288803. C325, C326

[8] H. C. Öttinger. A model of dilute polymer solutions with hydrodynamic interaction and finite extensibility. I. Basic equations and series expansions. J. Non-Newtonian Fluid Mech., 26(2): 207-246, 1987. http://dx.doi.org/10.1016/0377-0257(87)80005-8. C327

[9] H. C. Öttinger. Stochastic Processes in Polymeric Fluids. Springer, Berlin, 1996. C323, C325

[10] R. Prabhakar and J. R. Prakash. Multiplicative separation of the influences of excluded volume, hydrodynamic interactions and finite extensibility on the rheological properties of dilute polymer solutions. J. Non-Newtonian Fluid Mech., 116:163-182, 2004. http://dx.doi.org/10.1016/S0377-0257(03)00155-1. C325

[11] R. Prabhakar, J. R. Prakash, and T.Sridhar. A successive fine-graining scheme for predicting the rheological properties of dilute polymer solutions. J. Rheol., 48:1251-1278, 2004. http://dx.doi.org/10.1122/1.1807841. C328 
[12] J. Rotne and S. Prager. Variational treatment of hydrodynamic interaction in polymers. J. Chem. Phys., 50:4831-4837, 1969. http://dx.doi.org/10.1063/1.1670977. C324

[13] P. E. Rouse. A theory of the linear viscoelastic properties of dilute polymer solutions of coiling polymers. J. Chem. Phys., 21(7):1271-1280, 1953. http://dx.doi.org/10.1063/1.1699180. C321

[14] Douglas E. Smith and Steven Chu. Response of flexible polymers to a sudden elongational flow. Science, 281:1335-1340, 1998. http://dx.doi.org/10.1126/science.281.5381.1335. C327, C328

[15] P. Sunthar and J. Ravi Prakash. Parameter free prediction of dna conformations in elongational flow by successive fine graining. Macromolecules, 38:617-640, 2005. http://dx.doi.org/10.1021/ma0359411. C326, C327, C329

[16] B. H. Zimm. Dynamics of polymer molecules in dilute solution: Viscoelasticity, flow birefringence and dielectric loss. J. Chem. Phys., 24(2):269-281, 1956. http://dx.doi.org/10.1063/1.1742462. C321 